\title{
Imaging Seeker Surrogate for IRCM evaluation
}

\author{
H.M.A. Schleijpen ${ }^{1}$, S.R. Carpenter ${ }^{2}$, B. Mellier ${ }^{3}$, A. Dimmeler ${ }^{4}$ \\ ${ }^{1}$ TNO-Defence, Security and Safety, P.O. Box 96864, 2509 JG The Hague The Netherlands, \\ ${ }^{2}$ DSTL / Sensors Dept, 1016A, Bldg A2, Farnborough Hants, GU14 0LX, UK, \\ ${ }^{3}$ CELAR, Division DIRAC, 35170 Bruz, France, \\ ${ }^{4}$ FGAN-FOM Gutleuthausstrasse 1, 76275 Ettlingen, Germany
}

\begin{abstract}
NATO-SCI-139 and its predecessor groups have more than a decade of history in the evaluation and recommendation of EO and IR Countermeasures against anti-aircraft missiles. Surrogate Seekers have proven to be a valuable tool for this work. The use of surrogate seekers in international co-operations has several advantages over the use of an operational seeker, which is in service or in development: 1) the system is flexible, allowing both hardware and software modifications to be made in order to test the effectiveness of specific IRCM techniques; 2) the seeker design is open every last parameter is available to the science team, allowing detailed, end-to-end validation of software models and simulations; 3) the availability of an unclassified seeker facilitates open discussions on CM issues between the participants in the NATO-group.

Testing of high intensity countermeasures (for example based on lasers) needs a system with realistic seeker optics, with proper representation of optical scatter in seeker optics, which differs from scatter in commercial infrared camera optics.

A technical description of the ISS is given: an overview of the optical design and the detector, the principle of the tracking software and the possibilities to implement alternative tracking algorithms in order to represent different threat CCM techniques. The ISS is built for use both in the laboratory and in the field. Finally, some experimental results will be presented.
\end{abstract}

Keywords: IR Countermeasures, anti-aircraft missiles, Surrogate Seekers, laboratory tests, field trials.

\section{INTRODUCTION}

The threat of electro-optically guided missiles, historically a significant problem in military operations, is increasingly sophisticated. Developments in infrared and electro-optical technology have lead to seekers with high resolution imaging capability. This capability combined with image processing can now discriminate conventional countermeasures (CM) from the platform to be protected. New Infrared Countermeasure (IRCM) concepts against imaging seekers have to exploit the weak points of imaging seekers. Examples of new IRCM concepts are pointed IR lamps or lasers, called DIRCM (Directed Infrared Countermeasures), or DIRCM combined with conventional flares. Developing countermeasure concepts and testing their effectiveness in simulations and field trials is the aim of the task group SCI139 under the NATO RTO SCI panel. Although the countermeasure concepts investigated may have a broader application, the group focuses on the application against anti-aircraft missiles.

NATO-SCI-139 and its predecessor groups has a successful track record of international collaboration in the evaluation and recommendation of advanced EO and IR countermeasure techniques. The current programme of work of SCI-139 includes the following tasks:

- Developments of countermeasures and countermeasure concepts against imaging seekers,

- Digital simulations of the effectiveness of countermeasures using engagement models of 2D imaging seekers,

- Exchange of information on hardware seeker emulators,

- Tests of the effectiveness of countermeasure concepts against an Imaging Seeker Surrogate (ISS) in laboratory and field tests.

Ric.schleijpen@tno.nl phone+31 70374 0045, fax +31 703740654

Technologies for Optical Countermeasures III, edited by David H. Titterton,

Proc. of SPIE Vol. 6397, 63970E, (2006) · 0277-786X/06/\$15 - doi: 10.1117/12.689602

Proc. of SPIE Vol. $639763970 \mathrm{E}-1$ 


\section{SURROGATE SEEKERS}

The last task involves the use of a Surrogate Seeker. Surrogate seekers have proven to be a valuable tool for the work of the group for testing of countermeasures (CM). The use of a seeker surrogate has several advantages over the use of operational seekers, either in service or under development:

- The seeker surrogate is unclassified, which facilitates open discussions on CM issues between the participants in the NATO-SCI-139 group. The use of an operational seeker would seriously limit the possibilities for exchange of information.

- The system is more flexible than a specific operational system, allowing both hardware and software modifications to be made, in order to test the effectiveness of specific IRCM techniques against a variety of seeker configurations.

- The seeker design is open - every parameter and all software source is available to the science team, allowing detailed, end-to-end validation of software models and simulations.

Based on the results achieved with a previous surrogate seeker (representing a Rosette Scan Seeker) and encouraged by the success of this simulator, operated as a group research tool, the group decided to pursue the development of an Imaging Seeker Surrogate (ISS).

\section{REQUIREMENTS FOR THE ISS}

The Imaging Seeker Surrogate (ISS) is designed as a tool to evaluate new CM against the next generation seekers, which will be using imaging devices. The need for the seeker simulator to be representative for future threats leads to a set of requirements. First of all the ISS should have a detection and acquisition performance equivalent or better than state-ofthe-art imaging infrared seekers, with target tracking dynamics appropriate for an agile air-to-air missile against fast jet targets. This translates in the characteristics summarised in table 1. The seeker housing should be subject to the same physical constraints in terms of space envelope.

Table 1:

Design aims for ISS performance.

\begin{tabular}{|l|l|}
\hline Optical pass band & $3.7-4.8 \mu \mathrm{m}$, \\
\hline NEI, & $1.6 \times 10^{-10} \mathrm{~W} / \mathrm{m}^{2}$ \\
\hline NETD & $<50 \mathrm{mK}$ \\
\hline Acquisition range & $>10 \mathrm{~km}$. \\
\hline
\end{tabular}

Much work on imaging seekers can be done with infrared (IR) cameras. New countermeasure concepts however, are likely to involve high intensity sources such as lasers. High intensity countermeasures will generate optical scatter in the sensor optics. Since the design of seeker optics differs from commercial infrared camera optics, a system with realistic seeker optics, in which optical scatter is properly represented, is needed.

The ISS is built for use both in the laboratory and in the field. The laboratory work includes hardware in the loop HWIL tests, which requires suitable dimensions and robustness of the seeker head. For use in field tests the ISS is protected against adverse weather conditions. Both for laboratory tests and field tests the ISS can be mounted on different tracking platforms.

In order to enable realistic seeker behaviour during tests, a real-time tracking capability is required. This includes real time image processing capability. At the same time, because the system is intended to be used as a research tool, the image processing and tracking capability is reprogrammable. The system is still required to run real-time, despite the compromises which have to be made to allow reprogramming. Also a data recording system is required in order to collect raw seeker image data for off-line analysis. The off-line analysis will allow to run the same event for different algorithm settings in order to investigate the robustness of the countermeasures for a variety of seeker and tracking concepts.

The off-line analysis also allows the development and tuning of new algorithms. Here, the seeker hardware work is linked with the digital simulation work. On the one hand the results from laboratory and field tests with the ISS are used to validate digital simulation models. On the other hand the digital simulations will assist in developing real-time tracking algorithms with more robust infrared counter-countermeasures (IRCCM). Currently the digital simulation work within NATO SCI-139 focuses on the FLY-IN 2000 model [1]. The real-time processing algorithms of the ISS can be 
modelled in the high-level simulation code of FLY-IN 2000. This provides a solid basis for comparison of field trial and digital simulation studies.

The processing and control units are not integrated into the seeker head and are mounted in a separate rack. Overall control of both seeker and tracker functions is done from a dedicated host PC and appropriate Windows ${ }^{\mathrm{TM}}$ software.

\section{TECHNICAL DESCRIPTION OF THE ISS}

\subsection{Seeker head}

The two main components of the ISS are the seeker head and the seeker control unit. A design study for the seeker head was undertaken in 2000 to address the optical design in detail and to reduce the risk in the mechanical design. An outline diagram of the proposed system is shown in figure 1 .

The technique adopted for sightline control uses a pair of Risley prisms as shown in figure 2 just behind the dome. The prisms are rotated independently about a common axis to direct the sightline to the required direction. A discussion of the prism beam steering can be found in [2]. This refractive approach enables very rapid scanning using a simple and robust system, without the use of a mechanical gimbal.

It was apparent at the outset of the study that the chief technical challenge during the design process would be achromatisation of the optical train across the mid-infrared band. Where singlet germanium prisms had been suitable for beam steering in a long-wave design, larger doublet prisms are necessary, as a minimum, impacting on the mechanical design in the nose-cone region. The dome, originally hemispherical, was therefore pushed further forwards, adding problems of anamorphism and astigmatism to the system. A number of alternative configurations were explored, using a variety of materials chosen for their index and dispersion properties, to provide an optimised solution. A constraint on the study was that the electromechanical assembly (and therefore the interface to the main objective lens assembly) would remain unchanged. This was considered necessary to avoid additional risk and cost of both design and manufacture.

A reduced-curvature spherical-section dome was finally selected. Reducing the scan angle from $\pm 50^{\circ}$ to $\pm 40^{\circ}$ also helped reducing the prism thickness required and, in turn, the anamorphism. Reducing the optical bandpass also eased the control of aberrations introduced by dispersion of the prisms.

The design was also adapted to interface with the hardware in the loop (HWIL) simulation facility, to fit within the rollcage and connect to the both the sensor control unit and simulation control system.

Modifications to the mechanical design of the prism assemblies and associated structures were more significant than initially expected, as a result of the dome modifications. The housing structure was retained in the region of the drive electronics but now supports a larger dome module including the dome itself and its correcting optics. A further outer shroud was included in the design to improve final appearance. Figure 2 shows the seeker head with HWIL mounting flange.

Key features of the final design are as follows:

- Dome Sapphire or magnesium fluoride would normally be selected for a mid-IR dome that could withstand the supersonic missile flight environment. However, the ISS design uses a cheaper, zinc sulphide (Cleartran) material that is suitable for laboratory or ground test environments and is easier (and hence cheaper) to manufacture. This design has a diameter of $100 \mathrm{~mm}$ with radius of curvature of $100 \mathrm{~mm}$. The profile reduces astigmatic distortion of the image, and the profile of the seeker forebody has also been revised to match. Germanium and zinc sulphide correction lenses are fitted behind the dome into a screw-in module which, having zero optical power, may be removed without affecting image focus. This allows optical scatter to be assessed both with, and without, the dome (and perhaps, in the future, with an appropriately 'weathered' dome for comparison).

- Risley prisms The two prism modules are each constructed as dispersion-compensated doublets of silicon and germanium. Some degradation in spin acceleration (and hence optical beam-steering agility) results from their increased mass.

- Imaging lens The telescope assembly is an eight-element structure comprising both silicon and germanium elements. The final three objective lenses are fitted in a carrier fitted into the telescope housing via a fine thread and sufficient focusing adjustment is achieved by rotating the carrier. 
- Filter carousel A carousel arrangement having four apertures, allows any one of four optical components to be inserted in the optical path between the imaging lens and detector window. Currently these are three bandpass filters and one bandstop filter.

- Detector array The detector unit selected has a 256x256 cadmium-mercury-telluride (HgCdTe) array and incorporates an integrated Stirling closed-cycle cooling engine. An internal cold filter constrains its spectral response between 3.7-4.8 $\mu \mathrm{m}$. The seeker housing was adapted to contain a commercial IR camera electronics card set which provides all the functionality required to produce an image, including non-uniformity correction, digital and analogue video outputs. User programmability of 'stare' time, frame rate, correction method and bad pixel replacement options is via a serial interface and Windows ${ }^{\mathrm{TM}}$ software.

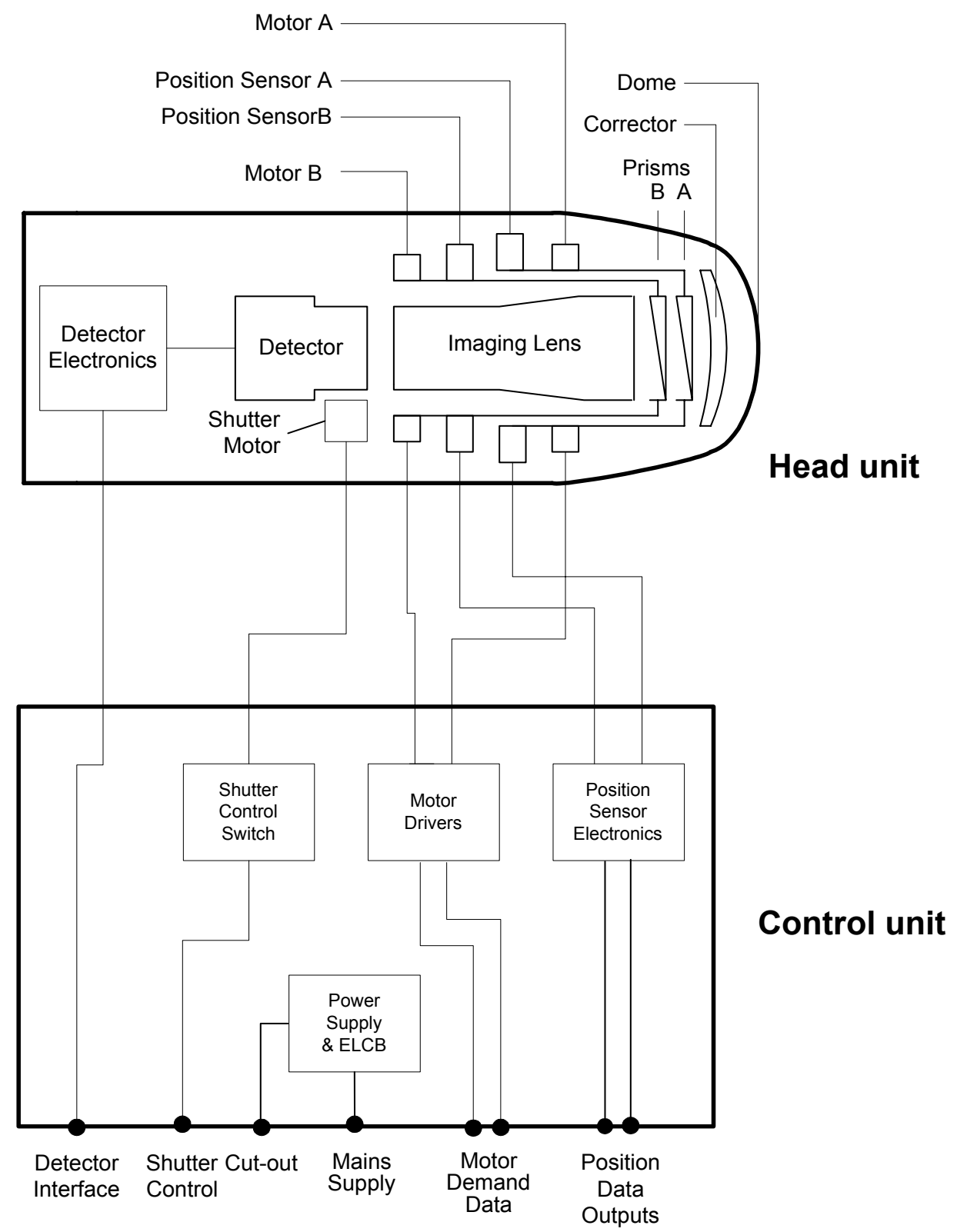

Figure 1: Schematic diagram of the ISS seeker head 


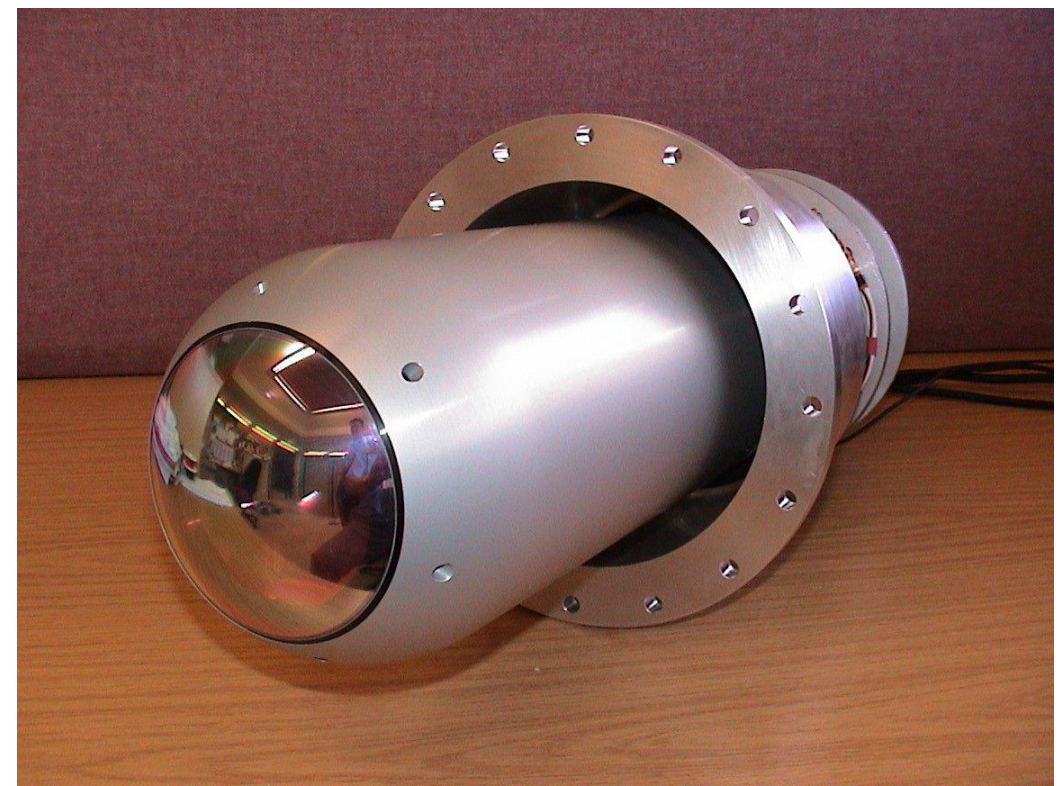

Figure 2: ISS Seeker Head

\subsection{Seeker Tracking and Control System}

A Configurable Logic Array Signal Processor (CLASP) has been selected for the seeker control tasks. It features a rackbased modular assembly of Altera ${ }^{\mathrm{TM}}$ field-programmable gate arrays (FPGAs) in a flexible and adaptable architecture for real-time signal processing; this design forms the basis of the signal processing and target tracking system for the ISS. In principle, a system similar to CLASP could be incorporated into the seekers of future missile systems. A reprogrammable processor of this type simplifies future upgrades quite dramatically, allowing the missile to be easily and cheaply re-configured, as new guidance and/or image processing techniques become available.

The current CLASP software incorporates a real-time quadrant-vector (Q-V) tracking algorithm coded into the FPGA architecture. Although originally developed as a baseline for long-wave seeker research and without countercountermeasure techniques specific to flares or jammers, it offers target-tracking capability that can be enhanced in the future to provide improved countermeasure resistance. Indeed, the complete flexibility in this area is a major strength of the system. The current version of the Q-V tracker has been ported to the FLY-IN 2000 simulation in for comparison tests. This represents a significant step in bridging the validation gap between digital simulations and real data from experiments and trials.

Overall control of CLASP and upload of FPGA code is from a host PC with Windows ${ }^{\mathrm{TM}}$ control software providing the user interface. Provision for manual sightline control via joystick, e.g., for initiating target acquisition, is incorporated. Standard CCIR video for the operator is provided and overlay graphics indicate vector search or quadrant tracker lock status and target size (derived from the area of the quadrant tracker box). An indication of the vector tracker's aim point is also shown, enabling the operator to ensure the system is locked onto the correct target if more than one target is present. An outer control loop provides additional azimuth and elevation demand signals for automatic tracking over large angles when the seeker is mounted on a motorised pan/tilt head. CLASP also incorporates a high-speed digital output interface suitable for driving an image data recorder (tape- or disk-based). For simplicity, key parametric data is inserted into the data stream on line one of every frame, allowing simple extraction on playback with appropriate software.

\subsection{System Specification}

The seeker specifications are summarised in table 2 .

The contracts and specifications for the design of the seeker head and the control units were supervised by DSTL Farnborough in the UK. 
Table 2: ISS specifications

\begin{tabular}{|c|c|}
\hline Mechanical (Seeker Head) & \\
\hline Forebody length & $38 \mathrm{~cm}$ (to rear mounting bulkhead) \\
\hline Forebody diameter & $15 \mathrm{~cm}$ \\
\hline Weight & $<25 \mathrm{~kg}$ \\
\hline Pitch axis inertia & $<1.4 \mathrm{~kg}-\mathrm{m} / \mathrm{s}$ \\
\hline Yaw axis inertia & $<1.4 \mathrm{~kg}-\mathrm{m} / \mathrm{s}$ \\
\hline \multicolumn{2}{|l|}{ Optical } \\
\hline Operating waveband & $3.7-4.8 \mu \mathrm{m}$ nominal \\
\hline Transmission & $>50 \%$ (aim) \\
\hline Field of view & $4.4^{\circ} \times 4.4^{\circ}$ \\
\hline Field of regard & $>0.7 \mathrm{rad}$ (radial) \\
\hline Optical resolution & Rayleigh limit $<0.2 \mathrm{mrad}$ \\
\hline (centre) & $>0.75$ of diffraction limit at $2 \mathrm{cy} / \mathrm{mrad}$ \\
\hline (edge FOV/FOR) & $>0.62$ of diffraction limit at $2 \mathrm{cy} / \mathrm{mrad}$ \\
\hline Focal length & $100 \mathrm{~mm}$ \\
\hline Focal ratio $(\mathrm{F} \#)$ & $\mathrm{F} / 2.8$ \\
\hline Detector angular subtense & $0.3 \mathrm{mrad}$ \\
\hline Optical sightline rate & $>5 \mathrm{rad} / \mathrm{s}$ each axis \\
\hline Optical sightline acceleration & $>3000 \mathrm{rad} / \mathrm{s}(\mathrm{aim})$ \\
\hline Angular position accuracy & $0.2 \mathrm{mrad}$ \\
\hline Dome material & ZnS Cleartran \\
\hline \multicolumn{2}{|l|}{ Detector } \\
\hline Type & $3-5 \mu \mathrm{m}$ focal plane array \\
\hline Material & CMT \\
\hline Array size & 256 by 256 elements \\
\hline Array pitch & $30 \mu \mathrm{m}$ \\
\hline Read-out technique & 'snapshot' method with variable stare time \\
\hline Cooling & Integrated Stirling cycle cooling engine \\
\hline \multicolumn{2}{|l|}{ System Performance } \\
\hline Frame rate & $>100 \mathrm{~Hz}$ \\
\hline System NETD & $<100 \mathrm{mK}$ \\
\hline Target acquisition range & $>10 \mathrm{~km}$ for fast jet (approx. 250W/sr) \\
\hline \multicolumn{2}{|l|}{ Environmental } \\
\hline Thermal range & $0^{\circ}$ to $30^{\circ} \mathrm{C}$ \\
\hline Relative humidity & $0 \%-50 \% \mathrm{RH}$ \\
\hline Maximum angular rate & $650 \%$ s (pitch \& yaw) \\
\hline Max angular acceleration & $1200 \% \mathrm{~s}^{2}$ (pitch \& yaw) \\
\hline \multicolumn{2}{|l|}{ Interfaces } \\
\hline Seeker head control & RS232/RS422 from CLASP \\
\hline Seeker head power & $24 \mathrm{~V} \mathrm{dc}$ from controller \\
\hline Seeker head video output & CCIR (analogue) for TV, RS422 to CLASP \\
\hline Risley prism position outputs & Seeker head to controller (analogue) \\
\hline Servo motor drives & Controller to seeker head (analogue) \\
\hline CLASP video output & CCIR (analogue) for TV monitor/recorder \\
\hline CLASP digital interface & RS422 digital output for data recording/replay \\
\hline CLASP platform control & RS232 from CLASP to motorised pan/tilt table \\
\hline Joystick & Allows manual control of seeker head \\
\hline PC extension cable $(20 \mathrm{~m})$ & Allows remote system control \\
\hline Power supply & $220-240 \mathrm{~V} 50 \mathrm{~Hz}$ ac \\
\hline
\end{tabular}

\section{FIELD TEST RESULTS}

The first field tests of the ISS took place in May 2004 and in April 2005. The main goal of these trials for the ISS was to check the operation of the system and to collect image sequences for further system improvement. A picture of the ISS at the trial on a tracking platform is given in figure 3. Figure 4 shows the ISS control station. 
The performance of the ISS is illustrated by the capability to track a commercial aircraft at $50 \mathrm{~km}$ at $30000 \mathrm{ft}$. A visible image and the corresponding infrared ISS image of this aircraft are shown in figure 5.

Example runs with a medium size transport aircraft deploying flares have shown that the QV-tracker is easily decoyed, as expected for a tracker with limited IRCCM capabilities.

The main conclusion for the ISS performance during these trials was that the system showed:

- good focus,

- good sensitivity,

- no EMI problems,

- no residual vignetting after NUC correction,

- $\mathrm{Q}-\mathrm{V}$ tracker functionality.

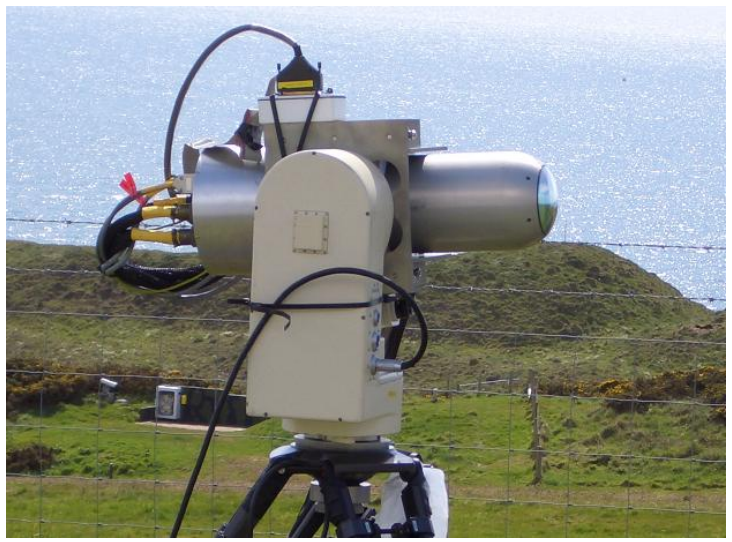

Figure 3: ISS on a tracking platform during ISS field tests
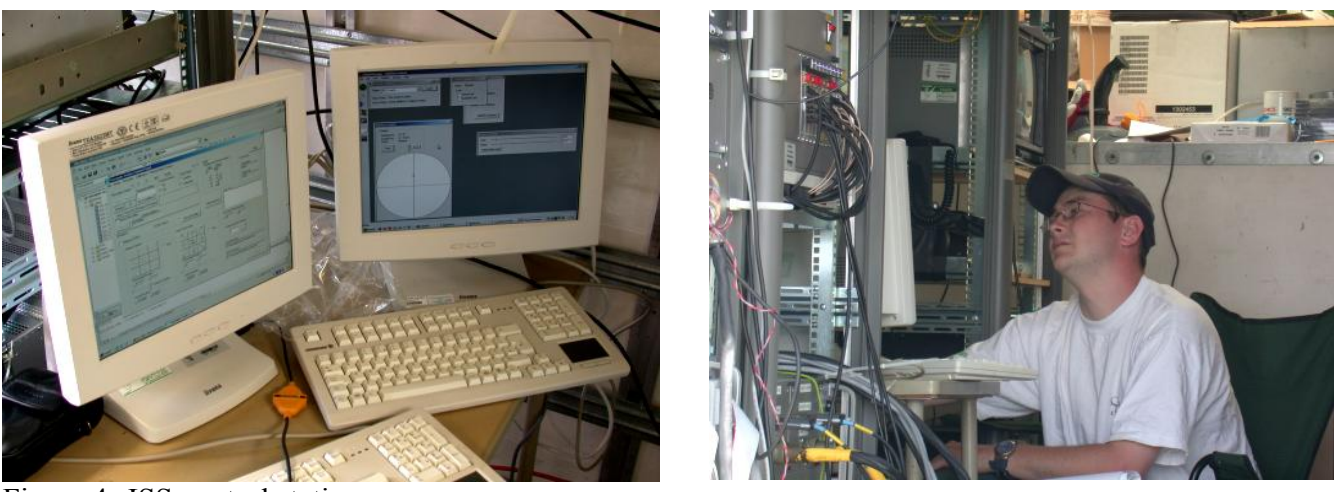

Figure 4: ISS control station
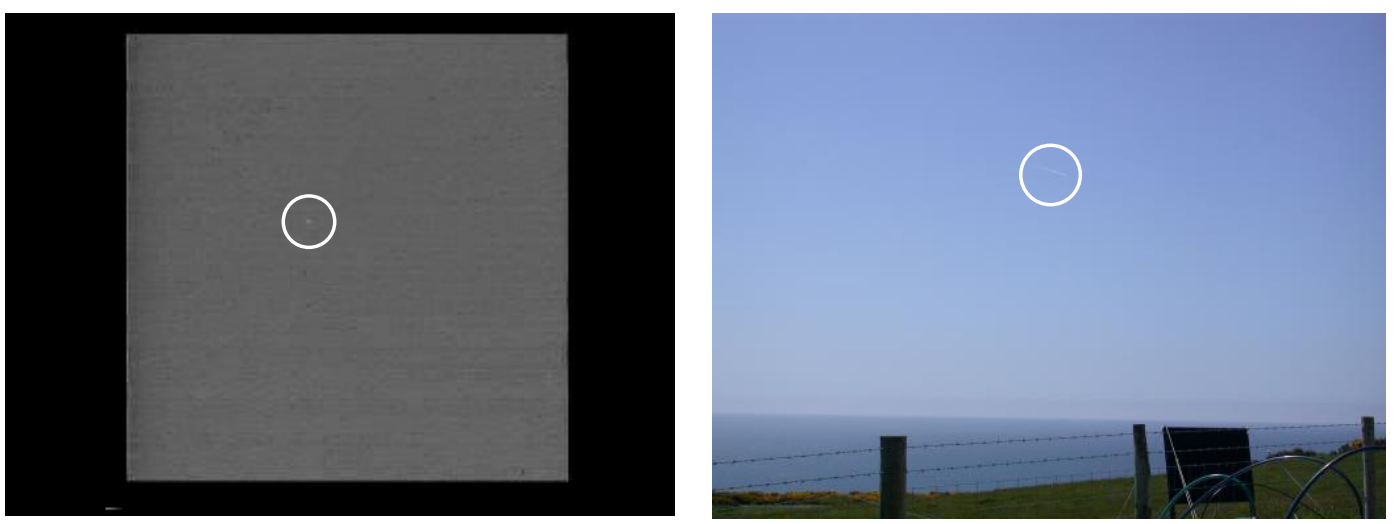

Figure 5: ISS Tracking of a commercial aircraft. ISS image (left) and corresponding visual image right. 


\section{OPTICAL CHARACTRISATION}

The measurements for the optical characterisation of the ISS were done at FGAN-FOM, Germany, using the conventional methods used for imaging systems.

\subsection{Noise}

Measurements of the noise level, measurements revealed that the noise level corresponds more to a NETD of $200 \mathrm{mK}$ than to the specified value of better than $50 \mathrm{mK}$ at $293 \mathrm{~K}$. The seeker noise is dominated by electronic noise. This high noise level is still subject of further checks. However, for the time being, this noise level, although higher than expected, is not expected to have a large effect on the results of high intensity laser work.

\subsection{Field of View (FOV) and Field of Regard (FOR)}

The FOV was measured directly and in addition was calculated from the recording of a MRTD-test-sample with a spatial frequency $0.5 \mathrm{mrad}^{-1}$ placed in the centre of the FOV. The FOV was found to be independent of the selected spectral filter. The measured results for the "boresight" position of the FOR as well as the nominal FOV (calculated from Pitch $(30 \mu \mathrm{m})$ and Focal Length $(100 \mathrm{~mm}))$ are presented in the upper half of table 3. Comparison shows a difference of approximately $10 \%$ for the horizontal and $7.5 \%$ for the vertical FOV.

Table 3: ISS FOV

\begin{tabular}{|l|c|c|}
\hline & \multicolumn{2}{|c|}{ FOV } \\
\hline & Horizontal & Vertical \\
\hline Boresight nominal & $4.4^{\circ}$ & $4.4^{\circ}$ \\
\hline Boresight direct measurement & $4.0^{\circ}$ & $4.1^{\circ}$ \\
\hline Boresight Calculated from MRTD test image & $4.0^{\circ}$ & $4.1^{\circ}$ \\
\hline Max Left & $5.7^{\circ}$ & $4.2^{\circ}$ \\
\hline Max Right & $5.8^{\circ}$ & $4.2^{\circ}$ \\
\hline Max Up & $5.8^{\circ}$ & $4.1^{\circ}$ \\
\hline Max Down & $5.8^{\circ}$ & $4.1^{\circ}$ \\
\hline
\end{tabular}

The Field of Regard (FOR) was measured to be approximately $\pm 43^{\circ}$ in the horizontal and approximately $\pm 43.5^{\circ}$ in the vertical plain. In addition, the FOV at maximum FOR was also measured. Figure 6 shows the recorded MRTD test sample in horizontal as well as vertical direction. It can clearly be seen how the test sample gets distorted at the different positions. In every image of Figure 6 the horizontal and vertical angles of the ISS-body are given, e. g. in the centre images the "boresight" position was $0^{\circ}$ horizontal and $5^{\circ}$ downwards ("boresight" inclination angle of the prisms of $5^{\circ}$ ). The calculated FOV values are listed in the lower part of table 3 . The seeker is operated in a $5^{\circ}$ off-boresight prism configuration to avoid the reduced agility of the tracking in the true boresight position due to the singularity for the counter rotating Risley prism steering in that position.

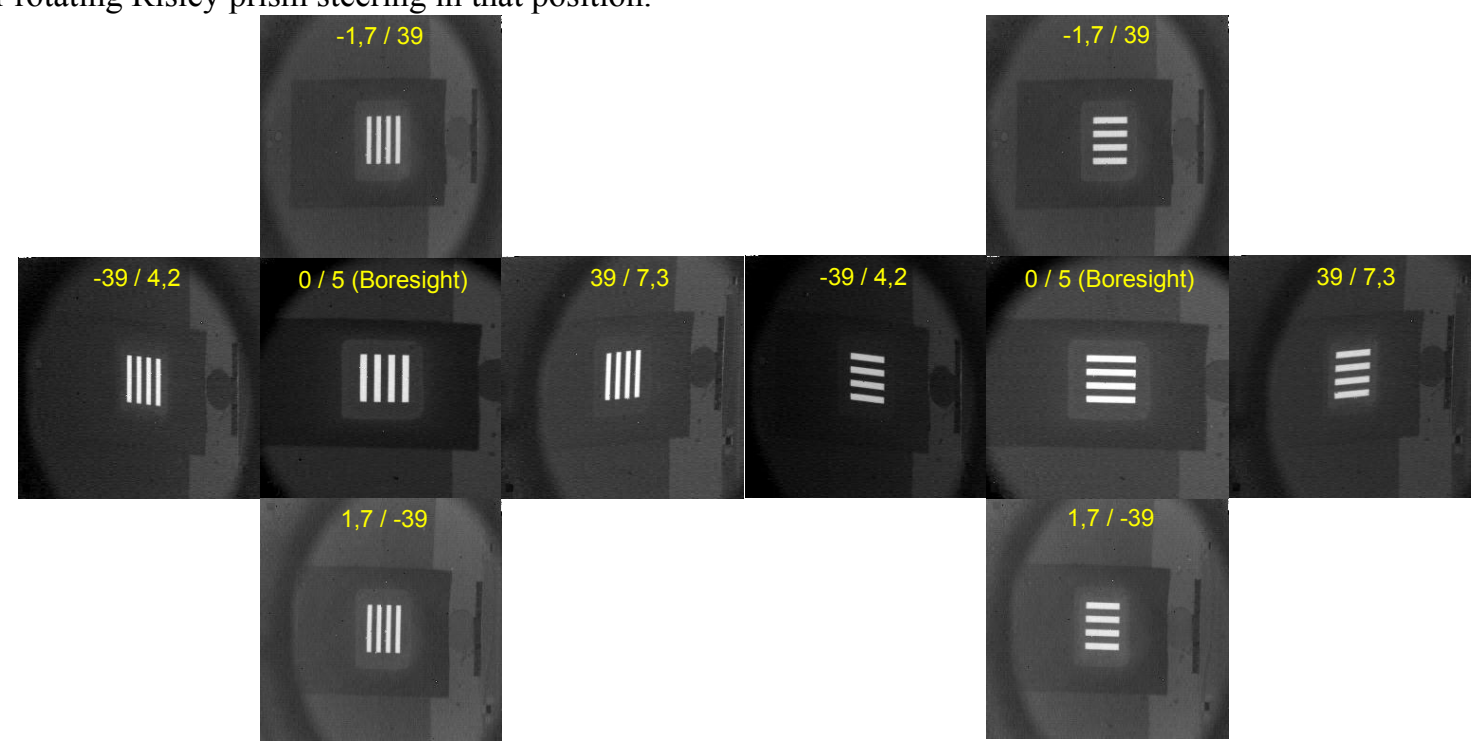

Figure 6: Appearance of MRTD-Test sample (horizontal right, vertical left) at different Field of Regard positions. 


\subsection{Modulation Transfer Function (MTF)}

The MTF was measured with the scanning edge method. For this method an edge (thin metal plate) is moved in front of a black-body and the signal of one detector element is recorded in dependence of the position of the plate. For the measurement the sampling distance is selected significantly smaller then the Instantaneous Field of View (IFOV) of the camera. The sampling distance was $0.04 \mathrm{mrad}$ and the temperature difference between black-body and plate was $2.5 \mathrm{~K}$. The measurement was conducted in horizontal as well as vertical direction for all four filters. In result one gets the signals in dependence of position, which are presented in Figure 7. The derivation of the edge function leads to the Line Spread Function (LSF), Figure 8, and the Fourier transformation of the LSF finally gives the MTF, Figure 9.

When the LSF is used to estimate the instantaneous field of view (IFOV) we obtain a value close to the detector angular subtence of $0.3 \mathrm{mrad}$.
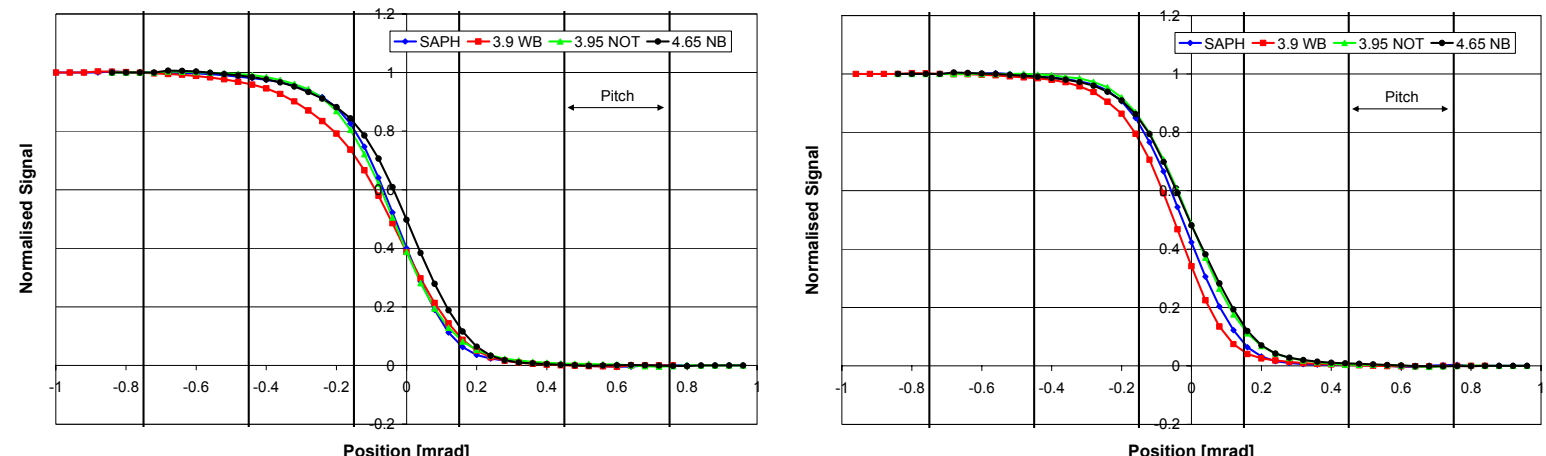

Figure 7: Horizontal (left) and vertical (right) Edge-functions for the four filters.
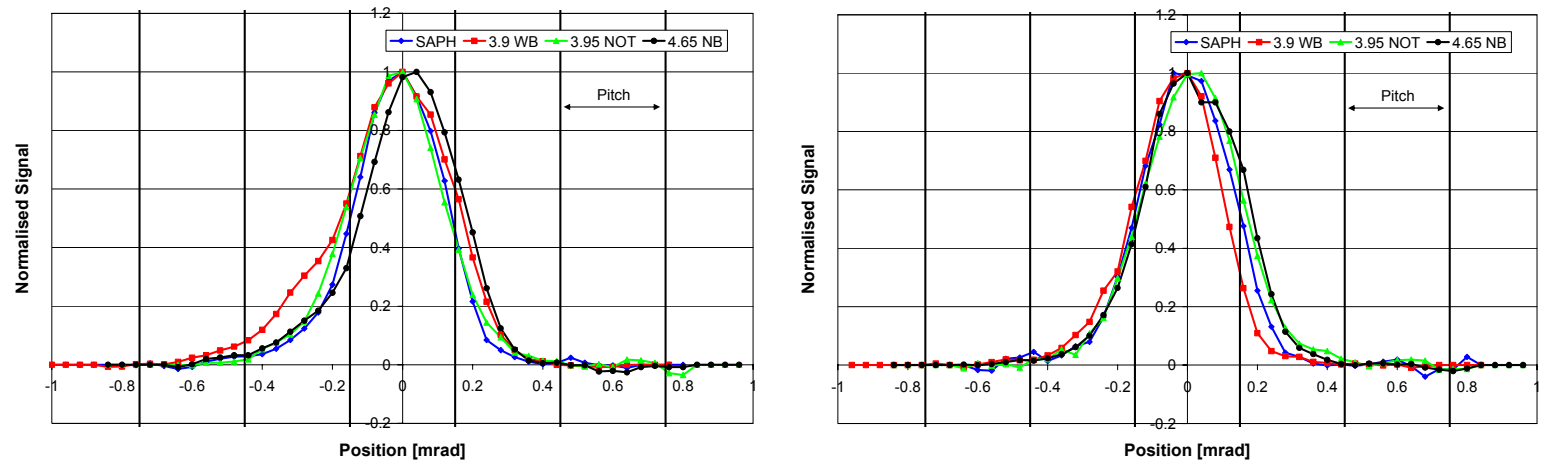

Figure 8: Horizontal (left) and vertical (right) LSF for the four filters. Additionally the pitch is given in graph.
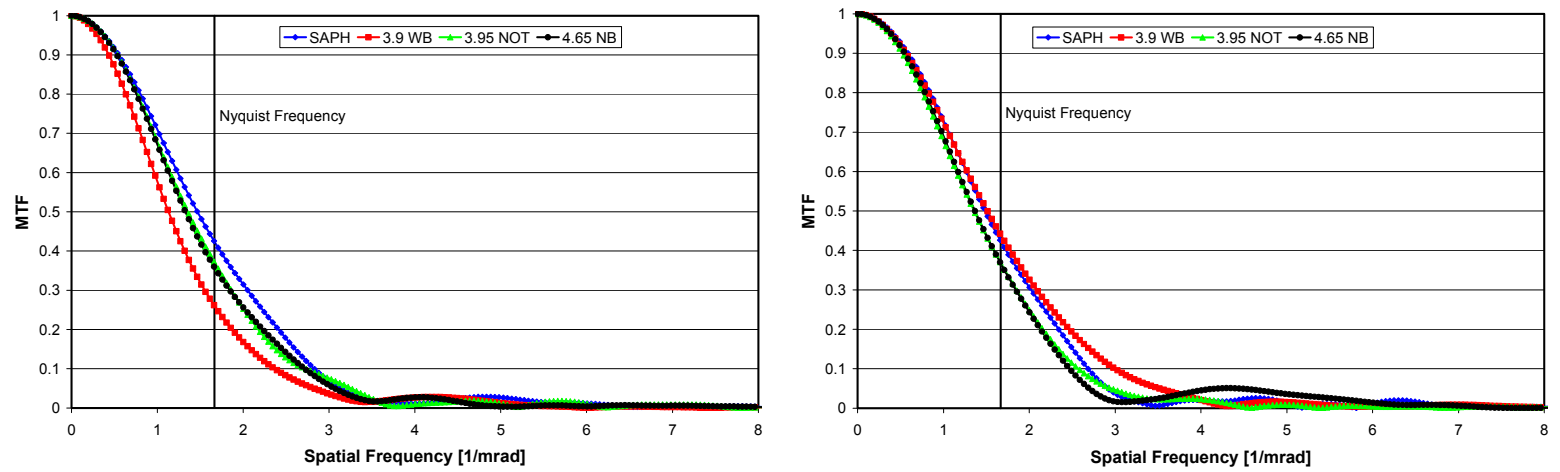

Figure 9: Horizontal (left) and vertical (right) MTF for the four filter. Additionally the Nyquist frequency is plotted in the graph. 


\section{LASER STIMULATION TESTS}

The tests characterizing the laser impact on the ISS image were performed at CELAR in France. These CELAR laser tests focused on the two following topics:

- effect of typical laser jamming waveforms on ISS image,

- effect of typical laser level of irradiance on ISS blooming spot.

The test set-up is illustrated in figure 10. The laser is a CW DF chemical laser modulated by an acousto-optic cell. This setup combined with calibrated attenuators enables generating controlled levels of laser irradiance of either $\mathrm{CW}$ emission or IR-jammer like emission (i.e. a laser pulse train). During all the laser tests, the ISS used $1 \mathrm{msec}$ integration time and $100 \mathrm{~Hz}$ frame rate. The laser irradiance levels on the seeker varied from $10^{+4} \mathrm{pW} / \mathrm{cm}^{2}$ to $10^{+7} \mathrm{pW} / \mathrm{cm}^{2}$. This range represents typical DIRCM laser irradiance levels on seekers, taking into account typical values for the laser power, the laser beam divergence and the engagement range. The upper level was selected to avoid and damage to the detector. For all the tests the laser was aligned along the seeker boresight in order to avoid ghost images which could disturb the interpretation of the test results. Although simulations have shown that ghost images can degrade the tracking in imaging seekers, it was decided to work on boresight only, since robust countermeasures should not depend on the effects of ghost images.
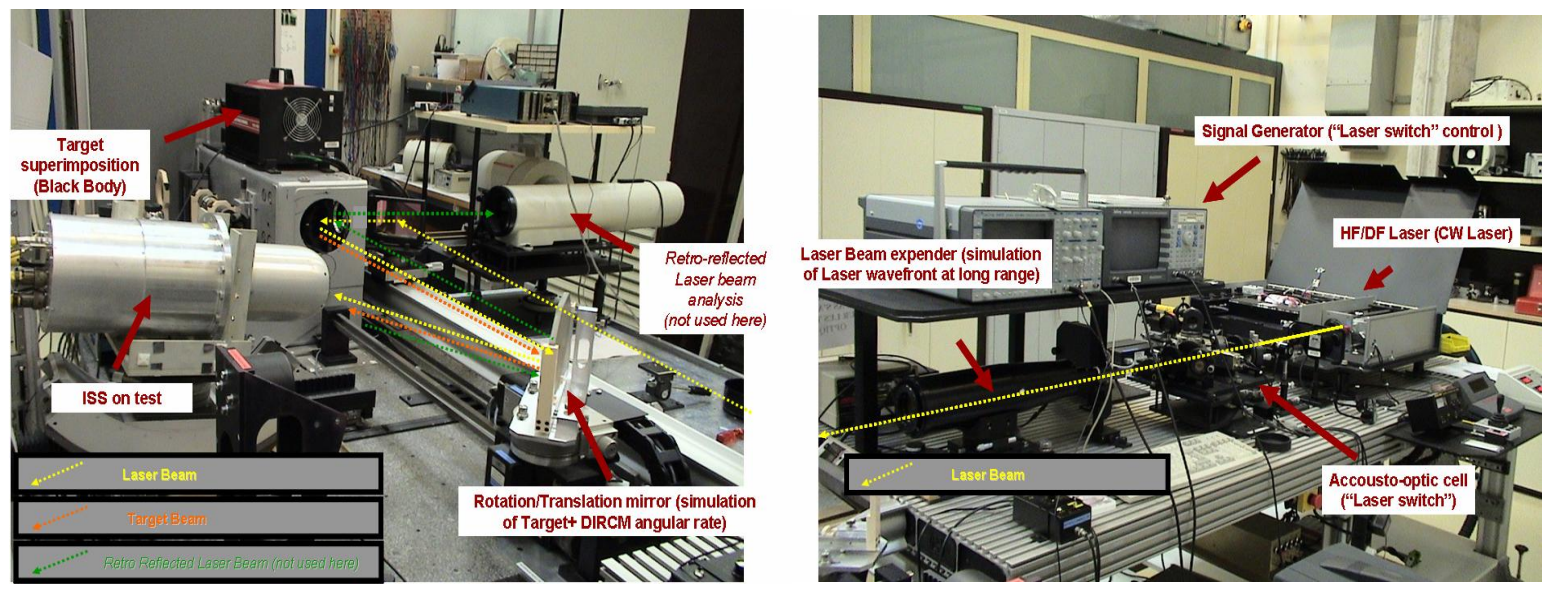

Figure 10: CELAR laser test set up

\subsection{Effect of typical laser jamming waveforms on ISS image:}

The temporal impact of laser jamming on seeker images is illustrated in figure 11. Figure 11 indicates the effects of modulated sources on an imaging seeker. The effects on the tracker differ very much from the jamming principles used against current non imaging seekers such as reticle seekers and scanning detector seekers. Depending on the phase and frequency difference of the jamming pulses and the seeker frame rate various (beating) effects can be achieved in imaging seekers. The actual effect of the jamming depends very much on the image interpretation by the specific tracking algorithm in combination with automatic gain effects.

\subsection{Effect of typical laser level of irradiance on ISS blooming spot:}

The impact of laser CW irradiance on the ISS images is illustrated in figure 12: These images show that:

- there is no "ghost image" for the tested levels of laser irradiance and for laser "on axis" stimulation (the weak spot in the images in figure 12 is the black body which is used for tracking),

- the blooming spots are relatively circular,

- $\quad$ as expected the size of blooming spot is increasing with laser level from 8 pixels for a laser irradiance of $10^{+5}$ $\mathrm{pW} / \mathrm{cm}^{2}$ up to a spot diameter of about 60 pixels for a laser irradiance of $10^{+7} \mathrm{pW} / \mathrm{cm}^{2}$ (diameter measured at half of saturation level).

During these tests, various laser waveforms were tested: $\mathrm{CW}$ and pulse trains at various frequency from 5 and $20 \mathrm{KHz}$ and various pulse durations between $5 \mu \mathrm{s}$ and $100 \mu \mathrm{s}$. Based on this variety of laser stimulations, the size of the observed laser spot diameter as a function of laser energy density is given in figure 13. This result is to be considered carefully because minimum laser pulse durations to be generated with the DF laser are between 1 and $5 \mu$ s $(5 \mu$ s to ensure a good temporal shape), which is about two orders of magnitude longer than OPO Based DIRCM laser pulses (typically a few tens of ns). 


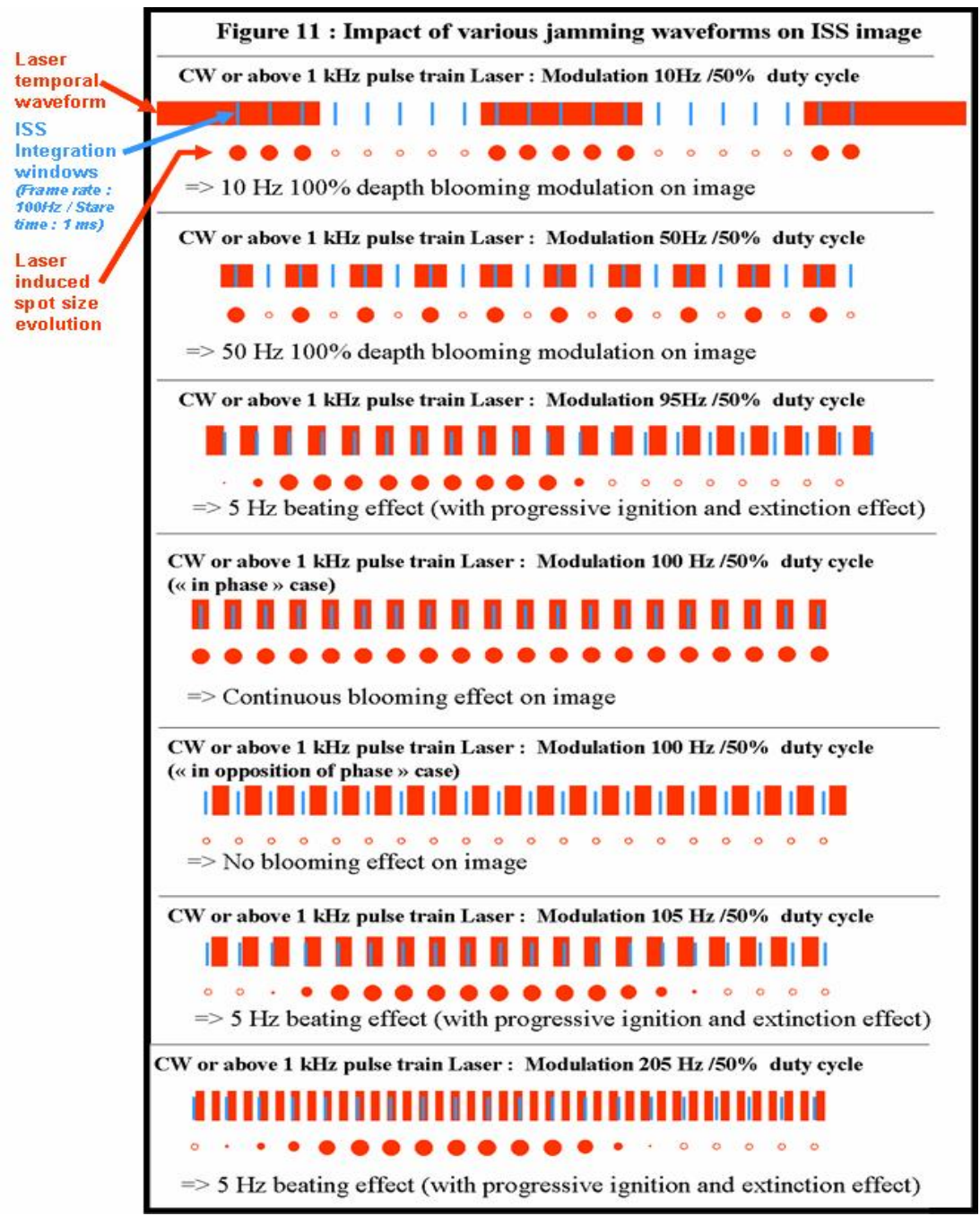

Figure 11: Impact of various jamming waveforms on the ISS image

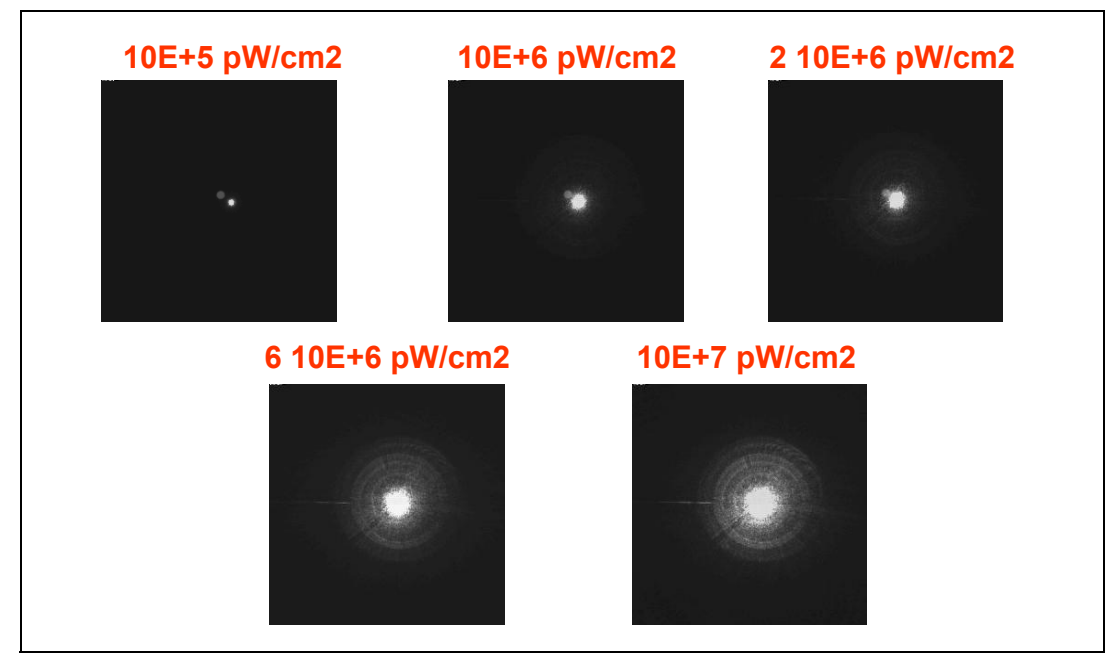

Figure 12: Impact of various CW laser irradiance levels on the ISS image 


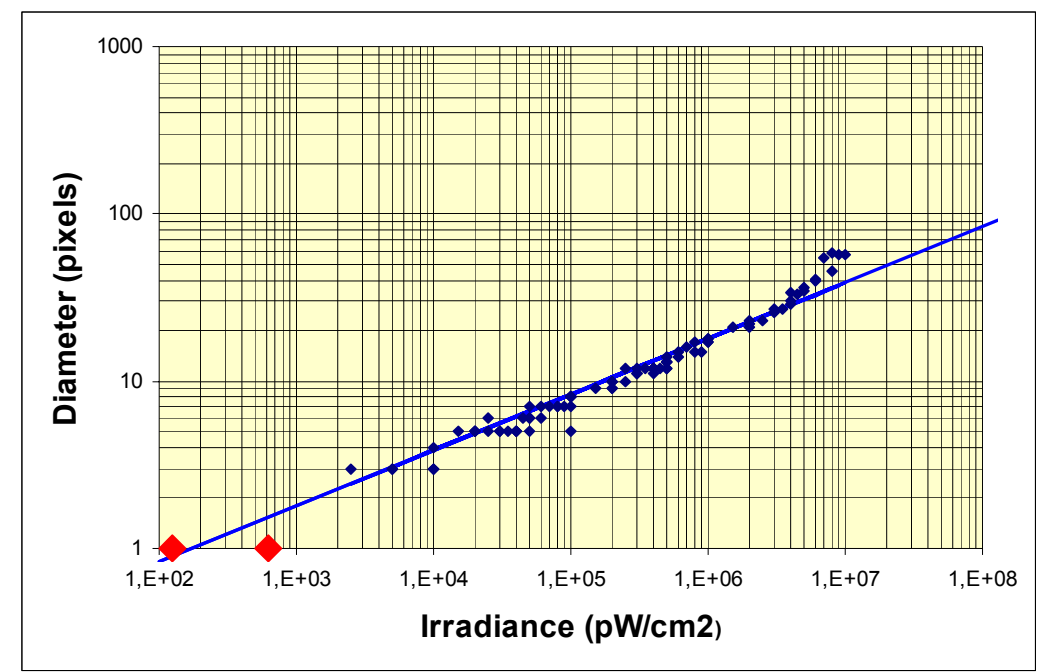

Figure 13: Observed laser spot diameter as a function of laser power density

In figure 13 the solid line indicates the empirical rule: increasing the lower power by a factor 8 will increase the saturated spot size by a factor 2 . Over the irradiance range covered by the experiments this rule seems to cover the experimental results. The maximum saturated spot size obtained in the experiments is 60 pixels in diameter. According to the experimental rule one would need 64 times more laser power (yielding a spot diameter of $4 \times 60=240$ pixels, to saturate almost the whole detector of 256x256 pixels. The experimental results at the higher irradiance levels seem to deviate from the experimental. Unfortunately these levels were not tested to avoid damage to the detector.

The large diamond marks indicate the irradiance level where a single pixel is just saturated. The two markers were derived following two different calculation methods. The first method uses the following assumptions on the seeker: $\mathrm{F} \# 2.8, \mathrm{f}=100 \mathrm{~mm}, \tau_{\text {optics }}=0.5$, fill factor 0.9 , quantum efficiency 0.65 , detector well capacity $3.710^{7}$ electrons. For a point source where all the energy hits the detector the saturation level will than be $625 \mathrm{pW} / \mathrm{cm}^{2}$. An alternative way of deriving the saturation level from the scene temperature where the array saturates $(385 \mathrm{~K})$ yields $124 \mathrm{pW} / \mathrm{cm}^{2}$. These values are as close to the empirical saturation rule as the experimental data. Current research is ongoing to verify a theoretical model for the increase of the size of the saturated area with increasing laser power. This model will link the power level where a single pixel is just saturated to the power levels needed for large area saturation.

\section{CONCLUSION}

The history of SCI-139 and its predecessor groups has clearly shown the benefits of working with a seeker simulator as a flexible research tool to evaluate the effectiveness of countermeasure concepts.

Through the acquisition of the Imaging Seeker Surrogate the group will have an up to date representation of current technology for imaging infrared seekers. It is a powerful tool which supports the countermeasure research programme in terms of laboratory and field tests and is supported by computer model validation.

In conjunction with its FLY-IN 2000 digital missile engagement simulation, this results in a generic countermeasure assessment toolset with which it will be able to design, test and demonstrate robust countermeasure techniques and strategies for future air platform protection.

\section{ACKNOWLEDGEMENT}

The authors would like to thank their colleagues of NATO SCI-139 for their support and discussions on this topic. The various contributions were sponsored by the MoDs from the Netherlands, Germany, France and the United Kingdom.

\section{REFERENCES}

1 L J Cox, S R Carpenter, M Batten, P A B Saddleton, "Modelling countermeasures to imaging infrared seekers", SPIE Vol 5615 October 2004.

2 B.D. Duncan, "Wide-angle achromatic prism beam steering for infrared countermeasure applications", Opt. Eng. 42(4), 1038-1047 April 2003. 